\title{
18. MIOCENE PLANKTONIC FORAMINIFERS AT DSDP SITE 289: DEPTH STRATIFICATION USING ISOTOPIC DIFFERENCES ${ }^{1}$
}

\author{
Joseph T. Gasperi² and James P. Kennett ${ }^{2}$
}

\begin{abstract}
Oxygen and carbon isotopic data for 13 planktonic foraminifers from the Miocene of DSDP Site 289 strongly support the idea that the species have a preferred depth habitat and that their depth relationships remained largely unchanged during the Miocene. However, the relative depth rankings of Globorotalia (Menardella) menardii and Globorotalia (Menardella) limbata changed from deep to intermediate and from deep to shallow, respectively, during the late Miocene between $\sim 9.9$ and $7.5 \mathrm{Ma}$.
\end{abstract}

\section{INTRODUCTION}

The primary purpose of this contribution is to present oxygen and carbon isotopic data for Miocene planktonic foraminifers from DSDP Site 289. A full discussion of the data is provided in Gasperi and Kennett (in press), which did not include the data, however, because of space limitations. The purposes of the study were to (1) establish relative rankings of species based upon $\delta^{18} \mathrm{O}$ and $\delta^{13} \mathrm{C}$ isotopic values, (2) determine how consistent these rankings were throughout the Miocene, and (3) identify the taxa, if any, that exhibit significant changes in rankings during the Miocene.

Modern planktonic foraminifers are depth stratified within the water column, their calcite tests commonly exhibiting oxygen and carbon isotopic compositional differences related to depth differences. The identification of depth stratification in fossil assemblages of planktonic foraminifers is useful in the study of the evolution of structure in the upper parts of the ocean. Previous studies of depth stratification among fossil planktonic foraminifers that have used $\delta^{18} \mathrm{O}$ values to establish depth rankings include Douglas and Savin (1978), Keller (1985), Savin et al. (1985), and Barrera et al. (1985). Berger et al. (1978) established a depth habitat ranking for latest Quaternary planktonic foraminiferal species from the Ontong Java Plateau.

\section{METHODS}

Sediment samples were washed with deionized water over a $74-\mu \mathrm{m}$ sieve and the fine fraction was saved. Planktonic foraminifers were picked from the $>250-\mu \mathrm{m}$ fraction if they were well preserved and present in sufficient numbers for isotopic analysis. The taxonomy of the planktonic foraminifers used in this study follows Kennett and Srinivasan (1983). Twenty-three of the most abundant species of planktonic foraminifers were picked from 51 samples at DSDP Site 289 for isotopic analysis. Picked samples were washed in methanol, sonified, and dried before being roasted under vacuum for $1 \mathrm{hr}$ at $375^{\circ} \mathrm{C}$. For each species, $10-40$ individuals (depending on mass) were run for isotopic analysis.

Isotopic analyses were conducted using a Finnigan Mat 251 mass spectrometer equipped with an automated carousel device located at the University of California, Santa Barbara. Values are given in delta notation as per mil deviations of the ${ }^{18} \mathrm{O} /{ }^{16} \mathrm{O}$ or ${ }^{13} \mathrm{C} /{ }^{12} \mathrm{C}$ ratio of the sample from that of the PDB standard. Isotopic analyses were related to PDB through repeated analyses of NBS-20 with values following Craig (1957) of $\delta^{18} \mathrm{O}$

\footnotetext{
${ }^{1}$ Berger, W.H., Kroenke, L.W., Mayer, L.A., et al., 1993. Proc. ODP, Sci. Results, 130: College Station, TX (Ocean Drilling Program).

${ }^{2}$ Department of Geological Sciences and Marine Science Institute, University of California, Santa Barbara, CA 93106, U.S.A.
}

$=-4.14 \%$ and $\delta^{13} \mathrm{C}=-1.06 \%$. Measurement of oxygen and carbon isotopic ratios have an analytical precision of $\pm 0.1 \%$.

The stratigraphic ranges of nine of the measured species are very restricted and the data are not included in this report. Also, the isotopic values of Globigerinita glutinata are highly variable and also are not included. The remaining 13 species are abundant and exhibit sufficiently long stratigraphic ranges for comparative studies (Tables 1 and 2).

\section{STRATIGRAPHY AND CHRONOLOGY}

The age model developed for DSDP Site 289 is based on paleomagnetically dated planktonic foraminiferal and calcareous nannofossil datum levels as compiled by Johnson and Nigrini (1985). The chronology follows the time scale of Berggren et al. (1985) whereby paleomagnetic Anomaly 5 is correlated with paleomagnetic Chron 11. The foraminiferal datums are compiled from Shipboard Scientific Party (1975) and Srinivasan and Kennett (1981a, 1981b), ages from Keller (1980), Berggren et al. (1985), and Barron et al. (1985), and magnetostratigraphic calibrations from Shipboard Scientific Party (1975), Keigwin (1982), Ryan et al. (1974), Miller et al. (1985), Poore et al. (1983), Berggren et al. (1983), and Barron et al. (1985). The calcareous nannofossil datums are compiled from Bukry (1975) and Shafik (1975), ages from Berggren et al. (1985) and Barron et al. (1985), and magnetostratigraphic calibrations from Haq et al. (1980) and Miller et al. (1985).

Samples from Site 289 were taken every $5-10$ m between 571 and $201 \mathrm{~m}$ below the seafloor (mbsf; Table 2), representing time intervals between 1 and $5 \times 10^{5} \mathrm{yr}$.

Table 2 shows the sample number, depth $(\mathrm{m})$, and age (Ma) of each of the 51 Miocene samples used in this study that range between $\sim 18.7$ and $5.8 \mathrm{Ma}$.

\section{RESULTS AND DISCUSSION}

Depth rankings (Table 1) have been determined for 13 Miocene $(\sim 18.7-5.8 \mathrm{Ma})$ planktonic foraminiferal species from the western equatorial Pacific (DSDP Site 289; Ontong Java Plateau; $2206 \mathrm{~m}$ present-day water depth; 2389 m paleowater depth at $\sim 22 \mathrm{Ma}$ ) based upon more than 600 oxygen and carbon isotopic analyses of their shells (Table 2).

Depth rankings based upon the carbon isotopic data do not always agree with those inferred from the oxygen isotopic data. The distribution of the oxygen isotopic data suggests that, as with modern species, no significant disequilibrium fractionation of the oxygen isotopes existed during calcification of any species. Thus, the discrepancies would appear to be a result of disequilibrium in the carbon isotopes. We propose that five inferred shallow-dwelling, spinose 
Table 1. Oxygen and carbon isotopic groupings and inferred depth rankings.

\begin{tabular}{|c|c|}
\hline$-18.7-7.5 \mathrm{Ma}$ & $-7.5-5.8 \mathrm{Ma}$ \\
\hline $\begin{array}{l}\text { Low } \delta^{16} \mathrm{O} \text { values and high } \delta^{13} \mathrm{C} \text { values: } \\
\text { Globigerinoides obliquus (S) } \\
\text { Globigerinoides sacculifer (S) } \\
\text { Globigerinoides immaturus (S) } \\
\text { Dentoglobigerina altispira }(\mathrm{S}) \\
\text { Globnquadrina baroemoenensis (S) }\end{array}$ & $\begin{array}{l}\text { Low } \delta^{18} \mathrm{O} \text { values and high } \delta^{13} \mathrm{C} \text { values: } \\
\text { Globigerinoides obliquus (S) } \\
\text { Globigerinoides sacculifer (S) } \\
\text { Globigerinoides immaturus (S) } \\
\text { Dentoglobigerina altispira (S) } \\
\text { Globoquadrina baroemoenensis (S) }\end{array}$ \\
\hline $\begin{array}{l}\text { Low } \delta^{18} \mathrm{O} \text { values and low } \delta^{13} \mathrm{C} \text { values: } \\
\text { Globorotalia (Jenkinsella) siakensis (S) } \\
\text { Globomtalia (Jenkinsella) mayeri (S) }\end{array}$ & $\begin{array}{l}\text { Low } \delta^{1 \mathrm{O}} \mathrm{O} \text { values and low } \delta^{13} \mathrm{C} \text { values: } \\
\text { Globorotalia (Menardella) limbata (S) }\end{array}$ \\
\hline $\begin{array}{l}\text { High } \delta^{1 \mathrm{X}} \mathrm{O} \text { values and low } \delta^{13} \mathrm{C} \text { values: } \\
\text { Globoquadrina dehiscens (D) } \\
\text { Globoquadrina venezuelana (D) } \\
\text { Globorotalia (Menardella) praemenardii (D) } \\
\text { Globorotalia (Menardella) menardii (D) } \\
\text { Globorotalia (Menardella) limbata (D) }\end{array}$ & $\begin{array}{l}\text { Intermediate } \delta^{18} \mathrm{O} \text { values and low } \delta^{13} \mathrm{C} \text { values: } \\
\text { Globorotalia (Menardella) menardii (1) } \\
\text { Globorotalia (Globorotalia) merotumida (I) } \\
\text { High } \delta^{18} \mathrm{O} \text { values and low } \delta^{13} \mathrm{C} \text { values: } \\
\text { Globoquadrina venezuelana (D) }\end{array}$ \\
\hline $\begin{array}{l}\text { otes: Shown are three groups of planktonic foran } \\
\text { upon the similarity of their oxygen and carb } \\
\text { foraminifers in the interval from }-7.5 \text { to } 5.8 \text {. } \\
\text { carbon isotopic values. Also shown are the } \\
\text { species ( } \mathrm{S}=\text { shallow, } \mathrm{I}=\text { intermediate, and } \mathrm{D}= \\
\text { based upon a number of assumptions: (1) eac } \\
\text { (2) water temperatures are highest at the surf } \\
\text { fractionation is temperature dependent so th } \\
\text { values relative to deeper dwelling species } \\
\text { foraminifers change vertically in the upper p. } \\
\text { higher in surface waters because of the highe } \\
\text { as respiration and decay of organic matter } \\
\text { species were ranked using (1) their relative } \\
\text { highest (deep). and (2) their relative carbon } \\
\text { (deep). However. much variability exists in th } \\
\text { between individual samples (Table 2). See G }\end{array}$ & $\begin{array}{l}\text { nifers in the interval from }-18.7 \text { to } 7.5 \text { Ma based } \\
\text { isotopic values and four groups of planktonic } \\
\text { a based upon the similarity of their oxygen and } \\
\text { erred depth rankings of planktonic foraminifer } \\
\text { eep). The isotopic depth ranking was established } \\
\text { species calcified its test in isotopic equilibrium; } \\
\text { e and decrease with depth: (3) oxygen isotopic } \\
\text { t shallower dwelling species have lower } \delta^{17} \mathrm{O} \\
\text { and (4) carbon isotopic values in planktonic } \\
\text { of the water column, such that } \delta^{13} \mathrm{C} \text { values are } \\
\text { biological productivity and decrease with depth } \\
\text { ycles }{ }^{12} \mathrm{C} \text {-rich } \mathrm{CO}_{2} \text { back into the water. Thus, } \\
\text { kygen isotopic values from lowest (shallow) to } \\
\text { otopic values from highest (shallow) to lowest } \\
\text { oxygen and carbon isotopic rankings of species } \\
\text { peri and Kennett (1992) for details. }\end{array}$ \\
\hline
\end{tabular}

species with high $\delta^{13} \mathrm{C}$ values (Globigerinoides immaturus, Globigerinoides sacculifer, Globigerinoides obliquus, Dentoglobigerina altispira, and Globoquadrina baroemoenensis) were hosts to large concentrations of algal symbionts. Their photosynthetic activities led to carbon isotopic fractionation and relatively high $\delta^{13} \mathrm{C}$ values of the host calcite (Spero and Williams, 1988). Depth rankings based upon $\delta^{18} \mathrm{O}$ and $\delta^{13} \mathrm{C}$ values for all measured species would agree if the high carbon isotopic values exhibited by these five species resulted solely from metabolically related isotopic fractionation.

From $\sim 18.7$ to $7.5 \mathrm{Ma}$, Globigerinoides obliquus, Globigerinoides sacculifer, Globorotalia (Jenkinsella) siakensis, Globorotalia (Jenkinsella) mayeri, Dentoglobigerina altispira, Globoquadrina baroemoenensis, and Globigerinoides immaturus were shallow-dwelling forms that are inferred to have had either moderately weak depth stratification from shallower to deeper as listed, or record slight variations in seasonal (temperature) distributions. Deep-dwelling forms include Globoquadrina dehiscens, Globorotalia (Menardella) limbata, Globorotalia (Menardella) menardii, Globoquadrina venezuelana, and Globorotalia (Menardella) praemenardii. No inferred intermediate-dwelling forms were recognized. The latest Miocene interval from $\sim 7.5$ to $5.8 \mathrm{Ma}$ marks a change in depth distribution of the Globorotalia (Menardella) group and the appearance for the first time of intermediate-dwelling forms. During this interval, shallow-dwelling forms include Globigerinoides obliquus, Globigerinoides sacculifer, Dentoglobigerina altispira, Globoquadrina baroemoenensis, Globorotalia (Menardella) limbata, and Globigerinoides immaturus. Intermediate-dwelling forms were Globorotalia (Menardella) menardii and Globorotalia (Globorotalia) merotumida, whereas Globoquadrina venezuelana was a deep-dwelling form.

The isotopic data for individual species strongly supports the idea that planktonic foraminifers have a preferred depth habitat and that their depth relationships remained largely unchanged during the Miocene. However, the relative depth rankings of Globorotalia (Menardella) menardii and Globorotalia (Menardella) limbata changed from deep to intermediate and from deep to shallow, respectively, during the late Miocene between $\sim 9.9$ and $7.5 \mathrm{Ma}$.

\section{ACKNOWLEDGMENTS}

The full paper of this report, but without the data, is that of Gasperi and Kennett (in press), which will be published under the auspices of the International Geological Correlation Project. We thank Professor Ryuichi Tsuchi, of Shizuoka University, for his enthusiastic and effective leadership of studies of Pacific Neogene stratigraphy through IGCP-246. This research was supported by NSF Grant No. DPP89-11554 (Division of Polar Programs) and OCE87-13391 (Submarine Geology and Geophysics).

\section{REFERENCES}

Barrera, E., Keller, G., and Savin, S.M., 1985. Evolution of the Miocene ocean in the eastern North Pacific as inferred from oxygen and carbon isotope ratios of foraminifera. Mem.-Geol. Soc. Am., 163:83-102.

Barron, J.A., Nigrini, C.A., Pujos, A., Saito, T., Theyer, F., Thomas, E., and Weinrich, N., 1985. Synthesis of biostratigraphy; central equatorial Pacific, Deep Sea Drilling Project, Leg 85: refinement of Oligocene to Quaternary biochronology. In Theyer, F., Mayer, L., et al., Init. Repts. DSDP, 85: Washington (U.S. Govt. Printing Office), 905-934.

Berger, W.H., Killingley, J.S., and Vincent, E., 1978. Stable isotopes in deep-sea carbonates: Box Core ERDC-92, West Equatorial Pacific. Oceanol. Acta, 1:203-216.

Berggren, W.A., Aubry, M.P., and Hamilton, N., 1983. Neogene magnetobiostratigraphy of DSDP Site 516 (Rio Grande Rise, South Atlantic). In Barker, P.F., Carlson, R.L., et al., Init. Repts. DSDP, 72: Washington (U.S. Govt. Printing Office), 675-713.

Berggren, W.A., Kent, D.V., and Van Couvering, J., 1985. The Neogene: Part 2, Neogene geochronology and chronostratigraphy. In Snelling, N.J. (Ed.), The Chronology of the Geological Record. Geol. Soc. London Mem., 10:211-260.

Bukry, D., 1975. Phytoplankton stratigraphy, southwest Pacific, Deep Sea Drilling Project, Leg 30. In Andrews, J.E., Packham, G., et al., Init. Repts. DSDP, 30: Washington (U.S. Govt. Printing Office), 539-548.

Craig, H., 1957. Isotopic standards for carbon and oxygen and correction factors for mass spectrometric analysis of carbon dioxide. Geochim. Cosmochim. Acta, 12:133-149.

Douglas, R.G., and Savin, S.M., 1978. Oxygen isotopic evidence for the depth stratification of Tertiary and Cretaceous planktonic foraminifera. Mar. Micropaleontol., 3:175-196.

Gasperi, J.T., and Kennett, J.P., in press. Isotopic evidence for depth stratification and paleoecology of Miocene planktonic foraminifera: western equatorial Pacific DSDP Site 289. In Tsuchi, R. (Ed.), IGCP-246 Pacific Neogene Events in Time and Space (PANETS): Tokyo (Univ. of Tokyo Press).

Haq, B.U., Worsley, T.R., Burckle, L.H., Douglas, R.G., Keigwin, L.D., Jr., Opdyke, N.D., Savin, S.M., Somner, M.A., III, Vincent, E., and Woodruff, F., 1980. Late Miocene marine carbon isotope shift and synchroneity of some phytoplanktic biostratigraphic events. Geology, 8:427-431.

Johnson, D.A., and Nigrini, C.A., 1985. Synchronous and time-transgressive Neogene radiolarian datum levels in the equatorial Indian and Pacific Oceans. Mar. Micropaleontol., 9:489-523.

Keigwin, L.D., Jr., 1982. Neogene planktonic foraminifers from Deep Sea Drilling Project Sites 502 and 503. In Prell, W.L., Gardner, J.V., et al., Init. Repts. DSDP, 68: Washington (U.S. Govt. Printing Office), 269-288.

Keller, G., 1980. Middle to late Miocene planktonic foraminiferal datum levels and paleoceanography of the north and southeastern Pacific Ocean. Mar. Micropaleontol., 5:249-281.

- 1985. Depth stratification of planktonic foraminifers in the Miocene ocean. Mem.-Geol. Soc. Am., 163:177-195.

Kennett, J.P., and Srinivasan, M.S., 1983. Neogene Planktonic Foraminifera: A Phylogenetic Atlas: Stroudsburg, PA (Hutchinson Ross).

Miller, K.G., Aubry, C., Khan, M.J., Melillo, A.J., Kent, D.V., and Berggren, W.A., 1985. Oligocene-Miocene biostratigraphy, magnetostratigraphy and isotopic stratigraphy of the western North Atlantic. Geology, 13:257-261.

Poore, R.Z., Tauxe, L., Percival, S.F., Jr., LaBrecque, J.L., Wright, R., Peterson, N.P., Smith, C.C., Tucker, P., and Hsü, K.J., 1983. Late CretaceousCenozoic magnetostratigraphic and biostratigraphic correlations for the South Atlantic Ocean, Deep Sea Drilling Project Leg 73. In Hsü, K.J., LaBrecque, J.L., et al., Init. Repts. DSDP, 73: Washington (U.S. Govt. Printing Office), 645-655.

Ryan, W.B.F., Cita, M.B., Rawson, M.D., Burckle, L.H., and Saito, T., 1974. A paleomagnetic assignment of Neogene stage boundaries and the devel- 
opment of isochronous datum planes between the Mediterranean, the Pacific and Indian Oceans in order to investigate the response of the world ocean to the Mediterranean "salinity crisis." Riv. Ital. Paleontol., 80:631-688.

Savin, S.M., Abel, L., Barrera, E., Hodell, D., Kella, G., Kennett, J.P., Killingley, J., Murphy, M., and Vincent, E., 1985. The evolution of Miocene surface and near-surface marine temperatures: oxygen isotopic evidence. In Kennett, J.P. (Ed.), The Miocene Ocean: Paleoceanography and Biogeography. Mem.-Geol. Soc. Am., 163:49-82.

Shafik, S., 1975. Nannofossil biostratigraphy of the southwest Pacific, Deep Sea Drilling Project, Leg 30. In Andrews, J.E., Packham, G., et al., Init. Repts. DSDP, 30: Washington (U.S. Govt. Printing Office), 549-598.

Shipboard Scientific Party, 1975. Site 289. In Andrews, J.E., Packham, G., et al., Init. Repts. DSDP, 30: Washington (U.S. Govt. Printing Office), 231-398.
Spero, H.J., and Williams, D.F., 1988. Extracting environmental information from planktonic foraminiferal $\delta^{13} \mathrm{C}$ data. Nature, 335:717-719.

Srinivasan, M.S., and Kennett, J.P., 1981a. A review of Neogene planktonic foraminiferal biostratigraphy: applications in the equatorial and south Pacific. Spec. Publ.-Soc. Econ. Paleontol. Mineral., 32:395-432.

1981b. Neogene planktonic foraminiferal biostratigraphy and evolution: equatorial to Subantarctic, South Pacific. Micropaleontology, $6: 499-533$.

Date of initial receipt: 6 July 1992

Date of acceptance: 20 August 1992

Ms 130B-061 
Table 2. Ranked oxygen and carbon isotopic data in each sample for 13 species for which relative depth rankings have been assigned (Table 1).

\begin{tabular}{|c|c|c|c|c|c|c|}
\hline Sample & Depth (mbsf) & Age (Ma) & $\delta 180$ & Species & $813 \mathrm{C}$ & Species \\
\hline \multirow[t]{8}{*}{$22-2, \quad 13-15$} & 201.13 & 5.80 & -1.25 & Gs. sacculifer & 2.03 & Gs. sacculiter \\
\hline & & & -1.20 & Gs. obliquus & 1.92 & Dg. altispira \\
\hline & & & -1.14 & Gs. immaturus & 1.75 & Gs. immaturus \\
\hline & & & -1.04 & Gr. limbata & 1.70 & Gs. obliquus \\
\hline & & & -1.00 & Gq. baroemoenensis & 1.59 & Gq. baroemoenensis \\
\hline & & & -0.97 & Gr. merotumida & 1.11 & Gr. merotumida \\
\hline & & & -0.92 & Dg. altispira & 1.03 & Gr. limbata \\
\hline & & & -0.63 & Gq. venezuelana & 0.75 & Gq. venezuelana \\
\hline \multirow[t]{9}{*}{$23-1, \quad 104-106$} & 210.04 & 6.02 & -1.56 & Gs. sacculifer & 2.32 & Gs. sacculifer \\
\hline & & & -1.55 & Gs. immaturus & 1.98 & Dg. altispira \\
\hline & & & -1.36 & Gs. obliquus & 1.88 & Gs. immaturus \\
\hline & & & -1.25 & Gq. baroemoenensis & 1.77 & Gs. obliquus \\
\hline & & & -1.18 & Dg. altispira & 1.61 & Gq. baroemoenensis \\
\hline & & & -1.17 & Gr. limbata & 1.10 & Gr. merotumida \\
\hline & & & -1.10 & Gr. menardii & 1.05 & Gr. limbata \\
\hline & & & -1.06 & Gr. merotumida & 1.04 & Gr. menardii \\
\hline & & & -0.75 & Gq. venezuelana & 0.76 & Gq. venezuelana \\
\hline \multirow[t]{9}{*}{$24-1, \quad 143-145$} & 219.93 & 6.26 & -1.46 & Gs. obliquus & 2.61 & Gs. sacculifer \\
\hline & & & -1.21 & Gr. limbata & 2.44 & Gq. baroemoenensis \\
\hline & & & -1.21 & Gs. sacculifer & 2.39 & Dg. altispira \\
\hline & & & -1.19 & Gs. immaturus & 2.30 & Gs. obliquus \\
\hline & & & -1.13 & Gq. baroemoenensis & 2.16 & Gs, immaturus \\
\hline & & & -1.11 & Dg. altispira & 1.73 & Gr. menardii \\
\hline & & & 0.99 & Gr. merotumida & 1.71 & Gr. limbata \\
\hline & & & -0.76 & Gr. menardii & 1.65 & Gr. merotumida \\
\hline & & & -0.73 & Gq. venezuelana & 1.33 & Gq. venezuelana \\
\hline \multirow[t]{9}{*}{$25-2,45-47$} & 229.95 & 6.50 & -1.33 & Gs. obliquus & 2.32 & Gs. sacculifer \\
\hline & & & -1.15 & Gs. sacculifer & 2.18 & Dg. altispira \\
\hline & & & -1.12 & Dg. altispira & 2.16 & Gq. baroemoenensis \\
\hline & & & -1.06 & Gq. baroemoenensis & 2.08 & Gs. obliquus \\
\hline & & & -1.04 & Gs. immaturus & 2.06 & Gs. immaturus \\
\hline & & & -1.01 & Gr. menardii & 1.64 & Gr. menardii \\
\hline & & & -0.99 & Gr. limbata & 1.61 & Gr. merotumida \\
\hline & & & -0.86 & Gr. merotumida & 1.58 & Gr. limbata \\
\hline & & & -0.49 & Gq. venezuelana & 1.40 & Gq. venezuelana \\
\hline \multirow[t]{6}{*}{$26-2,94-96$} & 239.94 & 6.85 & -1.37 & Gs. obliquus & 3.20 & Gs. sacculifer \\
\hline & & & -1.37 & Gs. immaturus & 3.00 & Dg. altispira \\
\hline & & & -1.34 & Gs. sacculifer & 2.90 & Gq. baroemoenensis \\
\hline & & & -1.25 & Gq. baroemoenensis & 2.74 & Gs. obliquus \\
\hline & & & -1.07 & Dg. altispira & 2.68 & Gs, immaturus \\
\hline & & & -0.75 & Gq. venezuelana & 1.86 & Gq. venezuelana \\
\hline \multirow[t]{6}{*}{$27-2, \quad 140-142$} & 249.90 & 7.20 & -1.14 & Gs. obliquus & 2.72 & Gs. sacculifer \\
\hline & & & -1.08 & Dg. altispira & 2.61 & Dg. altispira \\
\hline & & & -1.03 & Gs. sacculifer & 2.54 & Gq. baroemoenensis \\
\hline & & & -0.94 & Gq. baroemoenensis & 2.45 & Gs. obliquus \\
\hline & & & -0.83 & Gs. immaturus & 2.35 & Gs. immaturus \\
\hline & & & -0.59 & Gq. venezuelana & 1.61 & Gq. venezuelana \\
\hline
\end{tabular}


Table 2 (continued).

\begin{tabular}{|c|c|c|c|c|c|c|}
\hline Sample & Depth (mbsf) & Age (Ma) & 8180 & Species & $813 \mathrm{C}$ & Species \\
\hline \multirow[t]{9}{*}{$28-3,48-50$} & 260.00 & 7.46 & -1.26 & Dg. altispira & 2.68 & Gs. sacculifer \\
\hline & & & -1.12 & Gq. baroemoenensis & 2.55 & Dg. altispira \\
\hline & & & -1.08 & Gs. obliquus & 2.49 & Gs. immaturus \\
\hline & & & -1.01 & Gs. sacculifer & 2.43 & Gs. obliquus \\
\hline & & & .0 .95 & Gs. immaturus & 2.37 & Ga. baroemoenensis \\
\hline & & & -0.92 & Gr. limbata & 1.76 & Gr. limbata \\
\hline & & & -0.81 & Gr. merotumida & 1.68 & Gr. merotumida \\
\hline & & & -0.71 & Gr. menardii & 1.67 & Gr. menardii \\
\hline & & & -0.39 & Gq. venezuelana & 1.58 & Gq. venezuelana \\
\hline \multirow[t]{9}{*}{$29-3,104-106$} & 270.04 & 7.68 & -1.35 & Dg. altispira & 2.77 & Gs. sacculifer \\
\hline & & & -1.31 & Gs. obliquus & 2.64 & Gs. immaturus \\
\hline & & & -1.22 & Gs. immaturus & 2.58 & Dg. altispira \\
\hline & & & -1.04 & Gs. sacculifer & 2.44 & Gs. obliquus \\
\hline & & & -0.98 & Gq. baroemoenensis & 2.32 & Gq. baroemoenensis \\
\hline & & & -0.84 & Gq. venezuelana & 1.68 & Gr. merotumida \\
\hline & & & -0.71 & Gr. menardii & 1.66 & Gr. menardii \\
\hline & & 7 & -0.68 & Gr. limbata & 1.60 & Gr. limbata \\
\hline & & & -0.68 & Gr. merotumida & 1.41 & Gq. venezuelana \\
\hline \multirow[t]{5}{*}{$31-3, \quad 132-134$} & 289.32 & 8.10 & -1.30 & Gs. sacculifer & 2.78 & Gs. sacculifer \\
\hline & & & -1.14 & Gq. baroemoenensis & 2.28 & Dg. altispira \\
\hline & & & -1.02 & Dg. altispira & 2.22 & Gq. baroemoenensis \\
\hline & & & -0.87 & Gs. immaturus & 1.80 & Gr. menardii \\
\hline & & & -0.70 & Gr. menardii & 1.67 & Gr. limbata \\
\hline \multirow[t]{4}{*}{$32-5, \quad 130-132$} & 301.80 & 8.37 & -1.43 & Gq. baroemoenensis & 2.02 & Gs. immaturus \\
\hline & & & -1.08 & Dg. altispira & 1.90 & Dg. altispira \\
\hline & & & -1.03 & Gs. immaturus & 1.75 & Gq. baroemoenensis \\
\hline & & & -0.85 & Gr. menardii & 1.45 & Gr. menardii \\
\hline \multirow[t]{7}{*}{$33-5,8-10$} & 310.08 & 8.55 & -1.30 & Gs. sacculifer & 2.09 & Gs. sacculifer \\
\hline & & & -1.22 & Gs, obliquus & 1.92 & Gs. obliquus \\
\hline & & & -1.08 & Gs. immaturus & 1.90 & Gs. immaturus \\
\hline & & & -0.95 & Dg. altispira & 1.86 & Dg. altispira \\
\hline & & & -0.88 & Gq. baroemoenensis & 1.69 & Gq. baroemoenensis \\
\hline & & & -0.46 & Gq. venezuelana & 1.28 & Gr. menardii \\
\hline & & & -0.41 & Gr. menardii & 1.05 & Gq. venezuelana \\
\hline \multirow[t]{4}{*}{$34-2, \quad 113-115$} & 316.13 & 8.81 & -1.23 & Gq. baroemoenensis & 1.76 & Gq. baroemoenensis \\
\hline & & & -1.15 & Dg. altispira & 1.75 & Dg. altispira \\
\hline & & & -0.76 & Gr. menardii & 1.29 & Gr. menardii \\
\hline & & & -0.70 & Gq. venezuelana & 1.02 & Gq. venezuelana \\
\hline \multirow[t]{7}{*}{$34-6,96-98$} & 321.96 & 9.13 & -1.39 & Gs. obliquus & 2.21 & Gs. obliquus \\
\hline & & & -1.09 & Gs. immaturus & 2.15 & Dg. altispira \\
\hline & & & -1.06 & Dg. altispira & 2.07 & Gq. baroemoenensis \\
\hline & & & -0.75 & Gq. baroemoenensis & 2.05 & Gs. immaturus \\
\hline & & & -0.45 & Gr. menardii & 1.38 & Gr. menardii \\
\hline & & & -0.35 & Gr. limbata & 1.34 & Gr. limbata \\
\hline & & & .0 .25 & Gq. venezuelana & 1.14 & Gq. venezuelana \\
\hline
\end{tabular}


Table 2 (continued).

\begin{tabular}{|c|c|c|c|c|c|c|}
\hline Sample & Depth (mbsf) & Age (Ma) & $\delta 180$ & Species & $\delta 13 \mathrm{C}$ & Species \\
\hline \multirow[t]{5}{*}{$35-4,137-139$} & 328.87 & 9.50 & -1.43 & Gs. obliquus & 1.97 & Gs. obliquus \\
\hline & & & -1.33 & Gq. baroemoenensis & 1.94 & Dg. altispira \\
\hline & & & -1.31 & Dg. altispira & 1.68 & Gq. baroemoenensis \\
\hline & & & -0.75 & Gq. venezuelana & 1.28 & Gq. venezuelana \\
\hline & & & -0.69 & Gr. menardii & 1.10 & Gr. menardii \\
\hline \multirow[t]{9}{*}{$36-4,98-100$} & 337.98 & 9.90 & -1.22 & Gs. obliquus & 2.30 & Gs. obliquus \\
\hline & & & -1.12 & Gr. mayeri & 2.28 & Gs. immaturus \\
\hline & & & -0.94 & Dg. altispira & 1.98 & Dg. altispira \\
\hline & & & -0.92 & Gr. siakensis & 1.96 & Gq. baroemoenensis \\
\hline & & & -0.83 & Gq. baroemoenensis & 1.47 & Gr. mayeri \\
\hline & & & -0.72 & Gs. immaturus & 1.41 & Gq. dehiscens \\
\hline & & & -0.29 & Gq. dehiscens & 1.40 & Gr. siakensis \\
\hline & & & -0.10 & Gr. menardii & 1.34 & Gr. menardii \\
\hline & & & 0.07 & Gq. venezuelana & 1.29 & Gq. venezuelana \\
\hline \multirow[t]{6}{*}{$37-1,89-91$} & 342.89 & 10.31 & -1.33 & Gr. siakensis & 1.78 & Dg. altispira \\
\hline & & & -1.29 & Dg. altispira & 1.60 & Gs. obliquus \\
\hline & & & -1.27 & Gs. obliquus & 1.27 & Gq. venezuelana \\
\hline & & & -1.20 & Gr. mayeri & 1.19 & Gr. siakensis \\
\hline & & & -0.62 & Gq. venezuelana & 1.17 & Gr. menardii \\
\hline & & & .0 .46 & Gr. menardii & 1.07 & Gr. mayeri \\
\hline \multirow[t]{9}{*}{$37-6,47-49$} & 349.97 & 10.75 & -1.16 & Gs. obliquus & 2.32 & Gs. obliquus \\
\hline & & & $\because 0.98$ & Dg. altispira & $\cdot 2.20$ & Gs, immaturus \\
\hline & & & -0.91 & Gr. mayeri & $\cdot 1.58$ & Dg. altispira \\
\hline & & & $\cdot 0.88$ & Gs. immaturus & 1.44 & Gr. mayeri \\
\hline & & & $\because-0.72$ & Gq. baroemoenensis & 1.41 & Gr. siakensis \\
\hline & & & -0.63 & Gr. siakensis & 1.41 & Gr. menardii \\
\hline & & & -0.51 & Gq. venezuelana & 1.37 & Gr. praemenardii \\
\hline & & & -0.19 & Gr. menardii & 1.30 & Gq. venezuelana \\
\hline & & & 0.00 & Gr. praemenardii & $\cdot 1.23$ & Gq. baroemoenensis \\
\hline \multirow[t]{7}{*}{$38-4, \quad 106-108$} & 357.06 & 10.97 & $\because 1.36$ & Gr. mayeri & $\cdot 1.15$ & Gr. siakensis \\
\hline & & & $\because 1.18$ & Gr. siakensis & $\cdot 1.12$ & Gq. venezuelana \\
\hline & & & -0.68 & Gq. baroemoenensis & 1.02 & Dg. altispira \\
\hline & & & -0.66 & Gq. dehiscens & $\cdot 1.02$ & Gr. mayeri \\
\hline & & & -0.60 & Dg. altispira & 1.01 & Gq. dehiscens \\
\hline & & & $\because-0.37$ & Gq. venezuelana & $\cdot 1.00$ & Gq. baroemoenensis \\
\hline & & & -0.36 & Gr. praemenardii & 0.60 & Gr. praemenardii \\
\hline \multirow[t]{7}{*}{$39-2,48-50$} & 362.98 & 11.13 & -1.01 & Gr. mayeri & 2.60 & Gq. baroemoenensis \\
\hline & & & -1.00 & Dg. altispira & 2.57 & Gs. immaturus \\
\hline & & & -0.84 & Gr. siakensis & 2.34 & Dg. altispira \\
\hline & & & -0.73 & Gs. immaturus & 1.77 & Gq. venezuelana \\
\hline & & & -0.65 & Gq. baroemoenensis & 1.71 & Gr. praemenardii \\
\hline & & & -0.12 & Gr. praemenardii & 1.65 & Gr. siakensis \\
\hline & & & 0.01 & Gq. venezuelana & 1.59 & Gr. mayeri \\
\hline & & & & & & \\
\hline
\end{tabular}


Table 2 (continued).

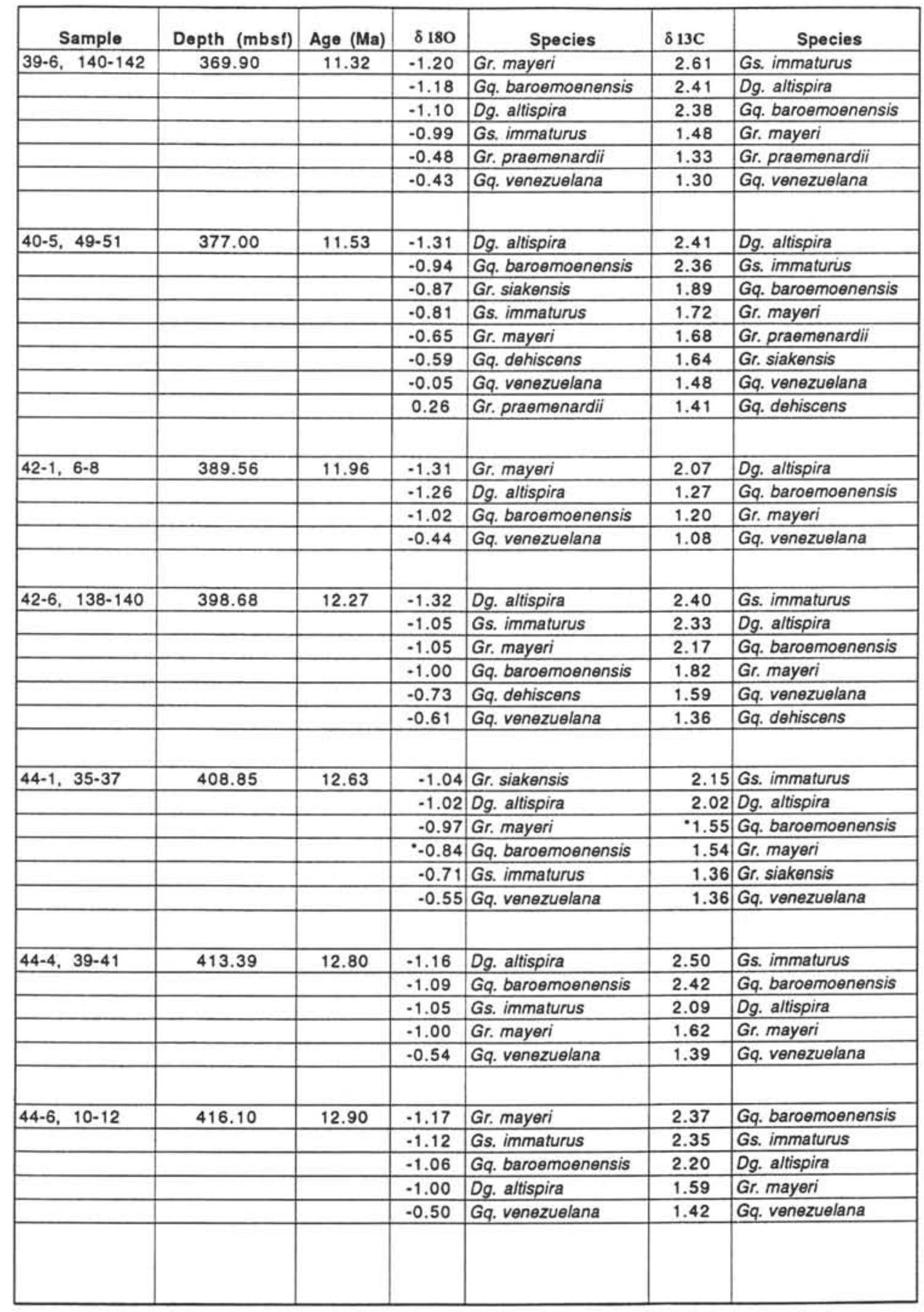


Table 2 (continued).

\begin{tabular}{|c|c|c|c|c|c|c|}
\hline Sample & Depth (mbsf) & Age (Ma) & 8180 & Species & $813 \mathrm{C}$ & Species \\
\hline \multirow[t]{6}{*}{$45-3, \quad 120-122$} & 422.20 & 13.12 & -1.40 & Gr. mayeri & 2.41 & Gs. immaturus \\
\hline & & & -1.29 & Gr. siakensis & 2.40 & Gq. baroemoenensis \\
\hline & & & -1.12 & Gs. immaturus & 2.03 & Dg. altispira \\
\hline & & & -1.05 & Dg. altispira & 1.58 & Gr. siakensis \\
\hline & & & -1.02 & Gq. baroemoenensis & 1.58 & Gr. mayeri \\
\hline & & & -0.66 & Gq. venezuelana & 1.40 & Gq. venezuelana \\
\hline \multirow[t]{4}{*}{$45-6,5-7$} & 425.55 & 13.23 & -1.32 & Gr. mayeri & 2.71 & Gq. baroemoenensis \\
\hline & & & -1.26 & Gq. baroemoenensis & 1.88 & Gr. mayeri \\
\hline & & & -1.25 & Gr. siakensis & 1.86 & Gr. siakensis \\
\hline & & & .0 .44 & Gq. venezuelana & 1.62 & Gq. venezuelana \\
\hline \multirow[t]{6}{*}{$46-2, \quad 148-150$} & 430.50 & 13.39 & -1.31 & Gr. siakensis & 2.54 & Gs. immaturus \\
\hline & & & -1.28 & Gr. mayeri & 2.44 & Gq. baroemoenensis \\
\hline & & & -1.20 & Gq. baroemaenensis & 2.23 & Dg. altispira \\
\hline & & & -1.16 & Gs. immaturus & 1.65 & Gr. siakensis \\
\hline & & & -1.16 & Dg. altispira & 1.61 & Gr. mayeri \\
\hline & & & .0 .28 & Gq. venezuelana & 1.50 & Gq. venezuelana \\
\hline \multirow[t]{5}{*}{$47-2, \quad 10-12$} & 438.60 & 13.66 & -1.42 & Gr. siakensis & 2.60 & Gq. baroemoenensis \\
\hline & & & -1.38 & Dg. altispira & 2.59 & Dg. altispira \\
\hline & & & -1.28 & Gr. mayeri & 1.86 & Gr. siakensis \\
\hline & & & -1.26 & Gq. baroemoenensis & 1.84 & Gr. mayeri \\
\hline & & & .0 .11 & Gq. venezuelana & 1.81 & Gq. venezuelana \\
\hline \multirow[t]{6}{*}{$47-4,54-56$} & 442.04 & 13.77 & -1.43 & Gr. mayeri & 2.98 & Dg. altispira \\
\hline & & & -1.35 & Dg. altispira & 2.94 & Gq. baroemoenensis \\
\hline & & & -1.28 & Gq. baroemoenensis & 2.83 & Gs. immaturus \\
\hline & & & -1.14 & Gr. siakensis & 2.07 & Gr. siakensis \\
\hline & & & -1.06 & Gs. immaturus & 1.98 & Gq. venezuelana \\
\hline & & & .0 .67 & Gq. venezulana & 1.98 & Gr. mayeri \\
\hline \multirow[t]{5}{*}{$48-1, \quad 140-142$} & 447.90 & 13.96 & -1.54 & Gr. mayeri & 2.80 & Gq. baroemoenensis \\
\hline & & & -1.31 & Gr. siakensis & 2.29 & Gs. immaturus \\
\hline & & & -1.27 & Gq. baroemoenensis & 1.93 & Gr. siakensis \\
\hline & & & -0.89 & Gs. immaturus & 1.87 & Gr. mayeri \\
\hline & & & -0.24 & Gq. venezuelana & 1.79 & Gq. venezuelana \\
\hline \multirow[t]{5}{*}{$48 \cdot 6,14-16$} & 454.15 & 14.17 & -1.69 & Gr. siakensis & 2.26 & Gq. baroemoenensis \\
\hline & & & -1.57 & Gr. mayeri & 2.13 & Gs. immaturus \\
\hline & & & -1.54 & Gq. baroemoenensis & 1.51 & Gr. siakensis \\
\hline & & & -1.11 & Gs. immaturus & 1.43 & Gq. venezuelana \\
\hline & & & -0.90 & Gq. venezuelana & 1.42 & Gr. mayeri \\
\hline \multirow[t]{5}{*}{$49-6, \quad 140-142$} & 464.90 & 14.53 & -1.23 & Gr. mayeri & 2.94 & Gq. baroemoenensis \\
\hline & & & .0 .99 & Gq. baroemoenensis & 2.50 & Gs. immaturus \\
\hline & & & -0.83 & Gs. immaturus & 2.08 & Gq. venezuelana \\
\hline & & & -0.71 & Gq. dehiscens & 1.95 & Gr. mayeri \\
\hline & & & -0.56 & Gq. venezuelana & 1.86 & Gq. dehiscens \\
\hline
\end{tabular}


Table 2 (continued).

\begin{tabular}{|c|c|c|c|c|c|c|}
\hline Sample & Depth (mbsf) & Age (Ma) & $\delta 180$ & Species & $813 \mathrm{C}$ & Species \\
\hline \multirow[t]{6}{*}{$50-2,48-50$} & 467.48 & 14.62 & -1.40 & Gr. siakensis & 2.77 & Dg. altispira \\
\hline & & & -1.33 & Dg. altispira & 2.59 & Gs. immaturus \\
\hline & & & -1.30 & Gr. mayeri & 2.49 & Gq. baroemoenensis \\
\hline & & & -1.22 & Gq. baroemoenensis & 2.08 & Gq. venezuelana \\
\hline & & & -0.88 & Gs. immaturus & 2.04 & Gr. mayeri \\
\hline & & & -0.78 & Gq. venezuelana & 2.00 & Gr. siakensis \\
\hline \multirow[t]{6}{*}{$50-6,133-135$} & 474.33 & 14.86 & -1.53 & Gr. mayeri & 3.16 & Gq. baroemoenensis \\
\hline & & & -1.41 & Gq. baroemoenensis & 3.16 & Dg. altispira \\
\hline & & & -1.40 & Gr. siakensis & 2.96 & Gs. immaturus \\
\hline & & & -1.34 & Dg. altispira & 2.38 & Gr. siakensis \\
\hline & & & -1.13 & Gs. immaturus & 2.38 & Gq. venezuelana \\
\hline & & & -0.64 & Gq. venezuelana & 2.34 & Gr. mayeri \\
\hline \multirow[t]{3}{*}{$51-4,48-50$} & 479.98 & 15.03 & -1.41 & Gq. baroemoenensis & 2.96 & Gq. baroemoenensis \\
\hline & & & -1.35 & Gr. mayeri & 1.96 & Gq. venezuelana \\
\hline & & & -0.60 & Gq. venezuelana & 1.90 & Gr. mayeri \\
\hline \multirow[t]{4}{*}{$52-2,42-44$} & 486.42 & 15.26 & -1.53 & Gq. baroemoenensis & 2.89 & Gq. baroemoenensis \\
\hline & & & -1.47 & Dg. altispira & 2.70 & Dg. altispira \\
\hline & & & -1.14 & Gs. immaturus & 2.44 & Gs. immaturus \\
\hline & & & -0.42 & Gq. venezuelana & 1.94 & Gq. venezuelana \\
\hline \multirow[t]{5}{*}{$52-6,129-131$} & 493.29 & 15.54 & -1.32 & Gr. siakensis & 3.02 & Gq. baroemoenensis \\
\hline & & & -1.30 & Gq. baroemoenensis & 2.94 & Dg. altispira \\
\hline & & & -1.27 & Gr. mayeri & 2.10 & Gr. siakensis \\
\hline & & & -1.14 & Dg. altispira & 2.07 & Gr. mayeri \\
\hline & & & -0.35 & Gq. venezuelana & 2.05 & Gq. venezuelana \\
\hline $53-4,122-124$ & 499.72 & 15.80 & \multicolumn{2}{|c|}{ NO DATA } & NO DATA & \\
\hline \multirow[t]{4}{*}{$54-1, \quad 128-130$} & 504.75 & 16.01 & -1.14 & Gg. baroemoenensis & 2.55 & Gs. immaturus \\
\hline & & & -1.01 & Gr. mayeri & 2.37 & Gq. baroemoenensis \\
\hline & & & -0.73 & Gs. immaturus & 1.98 & Gq. venezuelana \\
\hline & & & -0.17 & Gq. venezuelana & 1.91 & Gr. mayeri \\
\hline \multirow[t]{4}{*}{$54-3, \quad 58-60$} & 507.08 & 16.10 & -1.75 & Dg. altispira & 3.08 & Dg. altispira \\
\hline & & & -1.49 & Gq. baroemoenensis & 2.84 & Gq. baroemoenensis \\
\hline & & & -1.23 & Gq. venezuelana & 2.03 & Gq. venezuelana \\
\hline & & & .0 .94 & Gq. dehiscens & 1.97 & Gq. dehiscens \\
\hline \multirow[t]{4}{*}{$55-1,20-22$} & 513.20 & 16.35 & -1.59 & Dg. altispira & 2.81 & Dg. altispira \\
\hline & & & -1.41 & Gr. mayeri & 2.37 & Gq. baroemoenensis \\
\hline & & & -1.34 & Gq. baroemoenensis & 1.92 & Gq. venezuelana \\
\hline & & & -1.02 & Gq. venezuelana & 1.70 & Gr. mayeri \\
\hline
\end{tabular}


Table 2 (continued).

\begin{tabular}{|c|c|c|c|c|c|c|}
\hline Sample & Depth (mbsf) & Age (Ma) & $\delta 180$ & Species & $\delta 13 \mathrm{C}$ & Species \\
\hline \multirow[t]{4}{*}{$55-5,103-105$} & 520.03 & 16.63 & -1.78 & Dg. altispira & 2.55 & Dg. altispira \\
\hline & & & -1.57 & Gq. baroemoenensis & 2.19 & Gq. baroemoenensis \\
\hline & & & -1.12 & Gq. venezuelana & 1.69 & Gq. venezuelana \\
\hline & & & -0.90 & Gq. dehiscens & 1.38 & Gq. dehiscens \\
\hline \multirow[t]{2}{*}{$56-3,112-114$} & 526.62 & 16.90 & -1.25 & Gq. baroemoenensis & 2.23 & Gq. baroemoenensis \\
\hline & & & -0.79 & Gr. mayeri & 1.65 & Gr.mayeri \\
\hline \multirow[t]{8}{*}{$57-1,98-100$} & 532.98 & 17.16 & -1.26 & Gr. siakensis & 2.12 & Gs. sacculifer \\
\hline & & & -1.25 & Gr. mayeri & 1.99 & Gs. immaturus \\
\hline & & & -1.14 & Dg. altispira & 1.77 & Dg. altispira \\
\hline & & & -1.10 & Gs. sacculifer & 1.70 & Gq. baroemoenensis \\
\hline & & & -1.07 & Gq. baroemoenensis & 1.52 & Gr. siakensis \\
\hline & & & -0.90 & Gs. immaturus & 1.46 & Gq. venezuelana \\
\hline & & & -0.80 & Gq. venezuelana & 1.44 & Gr. mayeri \\
\hline & & & -0.71 & Gq. dehiscens & 1.18 & Gq. dehiscens \\
\hline \multirow[t]{3}{*}{$57-6,143-145$} & 540.90 & 17.48 & -1.11 & Gq. baroemoenensis & 2.26 & Gq. baroemoenensis \\
\hline & & & -1.05 & Gr. mayeri & 1.31 & Gr. mayeri \\
\hline & & & -0.73 & Gq. dehiscens & 1.28 & Gq. dehiscens \\
\hline \multirow[t]{5}{*}{$58-4, \quad 103-105$} & 547.03 & 17.79 & -1.31 & Gq. baroemoenensis & 2.09 & Dg. altispira \\
\hline & & & -1.22 & Dg. altispira & 1.80 & Gq. baroemoenensis \\
\hline & & & -1.05 & Gr. mayeri & 1.19 & Gq. venezuelana \\
\hline & & & -0.92 & Gq. dehiscens & 1.15 & Gr. mayeri \\
\hline & & & -0.30 & Gq. venezuelana & 1.13 & Gq. dehiscens \\
\hline \multirow[t]{2}{*}{$59-2, \quad 104-106$} & 553.54 & 18.14 & -1.38 & Gq. baroemoenensis & 1.69 & Gq. baroemoenensis \\
\hline & & & .0 .95 & Gr. mayeri & 1.24 & Gr. mayeri \\
\hline \multirow[t]{3}{*}{$60-1,98-100$} & 561.50 & 18.41 & -1.14 & Gr. mayeri & 1.54 & Gq. baroemoenensis \\
\hline & & & -1.00 & Gq. baroemoenensis & 1.52 & Gq. venezuelana \\
\hline & & & -0.72 & Gq. venezuelana & 1.43 & Gr. mayeri \\
\hline \multirow[t]{3}{*}{$61-1,95-97$} & 570.95 & 18.73 & -1.10 & Gr. mayeri & 1.81 & Gq. baroemoenensis \\
\hline & & & -1.04 & Gq. baroemoenensis & 1.37 & Gq. dehiscens \\
\hline & & & -0.81 & Gq. dehiscens & 1.33 & Gr. mayeri \\
\hline
\end{tabular}

DSDP Site 289 samples listed in meters below seafloor and by age. Data with an asterisk $\left({ }^{*}\right)$ are averages of two samples. 